\title{
miR-3662 suppresses cell growth, invasion and glucose metabolism by targeting HK2 in hepatocellular carcinoma cells
}

\author{
J. YE $E^{1, *}, \mathrm{X} . \mathrm{XIAO}^{2, *}, \mathrm{Y} . \mathrm{HAN}^{2}$, D. FAN ${ }^{2}$, Y. ZHU ${ }^{2, *}$, L. YANG ${ }^{2, *}$ \\ ${ }^{1}$ Department of Anesthesiology, Zhuhai Maternal and Child Health Hospital, Zhuhai, China; ${ }^{2}$ Department of Anesthesiology, The Fifth Affili- \\ ated Hospital of Sun Yat-Sen University, Zhuhai, China
}

*Correspondence: lijumoumi200610@yeah.net; pingmu658518@yeah.net

"Contributed equally to this work.

Received July 30, 2019 / Accepted September 29, 2019

\begin{abstract}
Hepatocellular carcinoma (HCC) is one of the most common malignancies with a rising incidence around the world. MicroRNAs (miRNAs) have been reported to play essential roles in the progression of HCC. However, the precise mechanism of miR-3662 in the HCC process remains poorly understood. This study was aimed to determine the regulatory network of miR-3662 and hexokinase 2 (HK2) in HCC. Quantitative real-time polymerase chain reaction (qRT-PCR) was performed to detect miR-3662 expression. Cell proliferation and invasion were measured by Cell Counting Kit-8 (CCK-8) assay and Transwell assay, respectively. Glucose consumption and lactate production assays were used to detect glucose metabolism activity in HCC cells. The potential binding sites between miR-3662 and HK2 were predicted by TargetScan online software and the relationship between miR-3662 and HK2 was verified by luciferase report assay. The protein expression of HK2 was measured by western blot analysis. A xenograft tumor model was established to confirm the role of miR-3662 and HK2 in vivo. miR-3662 expression was downregulated in HCC tissues and cells, and it was reduced in hypoxia-induced HCC cells in a time-dependent manner. Overexpression of miR-3662 or knockdown of HK2 inhibited cell proliferation, invasion, and glucose metabolism in HCC cells, which could be reversed by upregulating HK2. Besides, HK2 was a direct target of miR-3662 in HCC cells, and hypoxia upregulated the expression of HK2. In addition, the upregulation of HK2 could abolish miR-3662 overexpression-induced inhibitory effects on tumor growth and glucose metabolism in vivo.
\end{abstract}

Key words: hepatocellular carcinoma, hypoxia, miR-3662, HK2, growth, invasion, glucose metabolism

Hepatocellular carcinoma (HCC) is one of the most common malignancies with a rising incidence worldwide and the third most frequent cause of cancer related death [1, 2]. In spite of considerable therapeutic advances that have been made in the prevention of HCC, including surgical techniques and medical therapy, the disease is still associated with a poor prognosis [3]. Thus, it's urgent to study the molecular mechanism underlying HCC in order to search for a new diagnostic indicator or therapeutic targets for HCC treatment.

MicroRNAs (miRNAs) are small endogenous non-coding about 22 nucleotides RNAs that regulate protein expression by binding to the 3 '-untranslated regions (3'-UTR) of their target genes $[4,5]$. It has been reported that miRNAs are involved in a wide range of biological and pathological processes, such as cell proliferation, apoptosis, autophagy, metastasis, and invasion [6]. Increasing numbers of evidence revealed that the aberrant expression of specific miRNAs was closely associated with tumorigenesis and progression in a variety of cancers, including $\operatorname{HCC}[7,8]$. miR-3662 has been found as a novel tumor suppressor in leukemogenesis [9]. But, the level of miR-3662 was upregulated in patients with lung cancer. Additionally, it has been reported that the level of miR-3662 was decreased in HCC [10]. However, the precise mechanism of miR-3662 involved in the progression of HCC remains poorly understood.

One of the key features of most tumors is regional hypoxia [11]. Hypoxia-inducible factor (HIF) is the most important transcriptional regulator that regulates cellular responses to hypoxia. Activation of HIF-1a is closely associated with angiogenesis, erythropoiesis and the regulation of key enzymes involved in aerobic glycolysis, thereby modulating critical processes required for the Warburg effect [12]. Warburg effect, also known as the aerobic glycolysis, consists of an increase in glucose uptake and conversion of pyruvate to lactate, regardless of oxygen availability [13, 
14]. A core hallmark of cancer cells is aerobic glycolysis, and cancer cells rely on glycolysis as the main source of energy compare with normal cells [15]. Hexokinase (HK) catalyzes the first step of glucose metabolism, converting glucose to glucose-6-phosphate (G6P) [16]. The HK family consists of four isoforms, HK1, 2, 3 and 4. HK2 is highly expressed in various cancer cells relative to normal cells, and its high expression is closely related to poor prognosis [17-19]. HK2 may possess biologic and prognostic significance in HCC, with tumor HK2 expression being a potential independent predictor of survival [20]. Increasing evidence has suggested that most miRNAs could exert their biological function via binding to their downstream target genes. However, there is no evidence in support of the interaction between miR-3662 and HK2, and the function and mechanism of HK2 and cellular metabolism in HCC are largely unknown.

In this study, the expression levels of miR-3662 in HCC tissues, HCC cells, and hypoxia-induced HCC cells were detected. Subsequently, we investigated the effect of miR-3662 on cell proliferation, invasion and glucose metabolism, and explored the regulatory network of miR-3662/HK2. Taken together, this study might provide a novel sight for the treatment of HCC.

\section{Patients and methods}

Clinical specimens. Twenty-five pairs of HCC tissues and corresponding adjacent normal tissues were obtained from Zhuhai Maternal and Child Health Hospital. All participants signed written informed consent and this study was approved by the Research Ethics Committee of Zhuhai Maternal and Child Health Hospital. All samples were immediately frozen in liquid nitrogen and stored in $-80^{\circ} \mathrm{C}$ freezer.

Cell culture and transfection. HCC cell line (PLC/PRF/5) was purchased from the American Type Culture Collection (ATCC, Manassas, MD, USA). HCC cell line (Huh7) and normal liver cell line (LO2) were purchased from Shanghai Institute of Cell Biology, Chinese Academy of Sciences (Shanghai, China). All cell lines were cultured in Dulbecco's modified Eagle medium (DMEM, Hyclone, Logan, Utah, USA) and supplemented with $10 \%$ fetal bovine serum (FBS; Gibco, Carlsbad, CA, USA) in an incubator with $5 \% \mathrm{CO}_{2}$ at $37^{\circ} \mathrm{C}$. For anoxic treatment, cells were placed in a hypoxia chamber with $1 \%$ oxygen.

miR-3662 mimic (miR-3662) and its matched negative control (miR-NC), inhibitor miR-3662 (in-miR-3662) and its matched negative control (in-miR-NC), siRNA against HK2 (si-HK2) and its matched negative control (si-NC), HK2 overexpression vector (HK2) and empty vector (pcDNA) were purchased from GenePharma Co. Ltd. (Shanghai, China). According to the manufacturer's protocol, Huh7 and $\mathrm{PLC} / \mathrm{PRF} / 5$ cells were transfected with $100 \mathrm{nM}$ oligonucleotides or $2 \mu \mathrm{g}$ of the vector using Lipofectamine 3000 (Invitrogen, Carlsbad, CA, USA).
Quantitative real-time polymerase chain reaction (qRT-PCR). Following the manufacturer's instructions, Trizol reagent (Invitrogen) was used to extract total RNA from tissue samples or cells. The first strand of complementary DNA (cDNA) was synthesized using TaqMan microRNA Reverse Transcription Kit (Applied Biosystems, Foster City, CA, USA). Subsequently, cDNA was diluted and used for qRT-PCR to detect the expression level of miR-3662 using SYBR green detection kit (Takara, Dalian, China) on ABI 7500 real-time PCR machine (Applied Biosystems) according to the amplification instructions. Bulge-loop ${ }^{\mathrm{tm}}$ miRNA qRT-PCR Primer Sets (one RT primer and a pair of qPCR primers for each set) specific for miR-3662 and U6 small RNA were designed by RiboBio (Guangzhou, China). The relative expression level of miR-3662 was evaluated with $2^{-\Delta \Delta \mathrm{Ct}}$ method and using U6 small RNA as an internal control.

Cell proliferation assay. Following the manufacturer's instructions, cell viability of Huh7 and PLC/PRF/5 was measured by the Cell Counting Kit-8 (CCK-8; Dojindo Laboratories, Kumamoto, Japan). Briefly, Huh7 and PLC/ $\mathrm{PRF} / 5$ cells $\left(2 \times 10^{4}\right.$ cells $\left./ \mathrm{ml}\right)$ were seeded in 96 -well plates $(100 \mu \mathrm{l} /$ well). The next day, the cells were transfected with miR-3662, si-HK2, miR-3662+HK2 or their matched controls. After transfection for $24 \mathrm{~h}, 48 \mathrm{~h}$, and $72 \mathrm{~h}$ respectively, $10 \mu \mathrm{l}$ of CCK-8 solution was added to each well. Then, the plates were incubated for $2 \mathrm{~h}$ at $37^{\circ} \mathrm{C}$. Cell viability was evaluated at $450 \mathrm{~nm}$ using a microplate reader (Bio-Teck, Winooski, VT, USA).

Cell invasion assay. For transwell invasion assay, the transfected Huh7 and PLC/PRF/5 cells $\left(2 \times 10^{4}\right.$ cells/well) in $150 \mu \mathrm{l} \mathrm{serum}$-free DMEM medium were seeded in the top chamber with precoated Matrigel membrane filter inserts with $8 \mu \mathrm{m}$ pores in 24-well plates (BD, San Jose, CA, USA), and $500 \mu \mathrm{l}$ of DMEM medium supplemented with $10 \%$ FBS was added to the lower chamber. After incubation for $24 \mathrm{~h}$, cells on the top surface that did not invade through the pores were wiped with a cotton swab. The invading cells on the lower surface of the membranes were fixed with $4 \%$ paraformaldehyde for $20 \mathrm{~min}$ and stained with $0.1 \%$ crystal violet solution (Sigma, St. Louis, MO, USA). Cell counting was performed under a microscope (Olympus, Tokyo, Japan) in five random fields.

Detection of glucose and lactate levels. Huh7 and PLC/ $\mathrm{PRF} / 5$ cells were seeded in 12 -well plates $\left(1 \times 10^{4}\right.$ cells/well $)$ and incubated for $24 \mathrm{~h}$ prior to transfection. At $24 \mathrm{~h}$ posttransfection, the culture medium was harvested and used to measure the concentration of glucose or lactate. The consumption of glucose and production of lactate were measured using a glucose assay kit (Sigma) and lactate assay kit (BioVision, Mountain View, CA, USA), respectively. The glucose consumption or lactate production was examined by a standard calibration curve performed under the same condition and reported in a microplate reader according to the manufacturer's instructions. The results were normalized to the total protein. 
Luciferase reporter assay. The possible binding sites of miR-3662 and HK2 were predicted by online software TargetScan. In order to verify the direct binding of miR-3662 to the target HK2, a luciferase reporter assay was performed. The 3'-UTR of HK2 containing wild-type or mutant binding sites of miR-3662 was amplified and cloned into the pmirGlO luciferase reporter vector (Ambion, Austin, TX, USA) to generate the HK2 3'-UTR-WT or HK2 3'-UTR-MUT, respectively. Following the protocols of manufacturer, the miR-3662 or miR-NC was co-transfected with WT or MUT luciferase reporter plasmids into Huh7 and PLC/PRF/5 cells using Lipofectamine 3000 (Invitrogen). $48 \mathrm{~h}$ later, cells were lysed and luciferase activities were detected using DualLuciferase Assay Kit (Promega, Madison, WI).

Western blot assay. Total proteins were extracted from Huh7 and PLC/PRF/5 cells using radio-immunoprecipitation assay (RIPA) lysis buffer (Sigma) with protease inhibitor (Sigma) for $20 \mathrm{~min}$ at $4^{\circ} \mathrm{C}$ followed by centrifugation at $12,000 \times \mathrm{g}$ for $15 \mathrm{~min}$ at $4^{\circ} \mathrm{C}$ to collect the supernatant. Subsequently, the proteins were quantified by bicinchoninic acid (BCA) Kit (Pierce Rockford, IL, USA). A total of $40 \mu \mathrm{g}$ protein was separated by $8 \%$ sodium dodecyl sulfate polyacrylamide gel electrophoresis (SDS-PAGE), and then transferred by electrophoresis onto polyvinylidene fluoride (PVDF) membranes (Millipore, Billerica, MA, USA). The membranes were blocked in Tris-buffered saline and $0.1 \%$ Tween 20 (TBST) butter with 5\% (w/v) non-fat milk for $2 \mathrm{~h}$ at room temperature, and then washed 3 times with TBST and then incubated overnight at $4{ }^{\circ} \mathrm{C}$ with primary antibodies against HK2 (1:1000, \#2106, Cell Signaling Technology, Beverly, MA, USA) or $\beta$-actin (1:1000, \#4970, Cell Signaling Technology). The membranes again were washed 3 times using TBST, followed by incubation with horseradish peroxidase-conjugated secondary antibodies (Sangon Biotech) at 1:4000 dilutions for $2 \mathrm{~h}$ at room temperature. Finally, the protein bands were identified using an enhanced chemiluminescence (ECL; Tanon, Shanghai, China) solution. The densitometry of the bands was examined by the ImageJ software.

In vivo experiment. Five weeks old female $\mathrm{BALB} / \mathrm{c}$ mice were purchased from Henan Experimental Animals Centre (Zhengzhou, China) and caged under specific pathogen free conditions. All experimental procedures were performed following the guidelines for the care and use of laboratory animals. And the procedures were approved by the committee of the Animal Research of Zhuhai Maternal and Child Health Hospital. All BALB/c nude mice were divided into 4 groups $\left(\mathrm{n}=7\right.$ /group) randomly. Huh7 cells $\left(1 \times 10^{6}\right)$ transfected with miR-3662, HK2 or miR-3662+HK2 were subcutaneously injected into either side of the flank of the nude mice. Tumor volumes were examined every $7 \mathrm{~d}$ with a caliper and calculated by the formula: volume $=\left(\right.$ length $\times$ width $\left.^{2}\right) / 2$. At $28 \mathrm{~d}$ post tumor implantation, all mice were sacrificed, and then tumors were removed and weighed. Tumors were harvested for subsequent glucose metabolism analysis.

Statistical analysis. Data were presented as mean \pm standard deviation (SD) from at least three individual experiments. The statistical differences between groups were analyzed by Student's $t$ test using GraphPad Prism version 6.0 software (GraphPad Software, San Diego California, USA). A p-value $<0.05$ was regarded as statistically significant.

\section{Results}

The miR-3662 expression was downregulated in HCC tissues and cells, and it was reduced in hypoxia-induced HCC cells. To explore the potential roles of miR-3662 in the progression of HCC, qRT-PCR was performed to explore the expression of miR-3662 in HCC tissues, HCC cells, and hypoxia-induced HCC cells. As shown in Figure 1A, the expression level of miR-3662 was drastically decreased
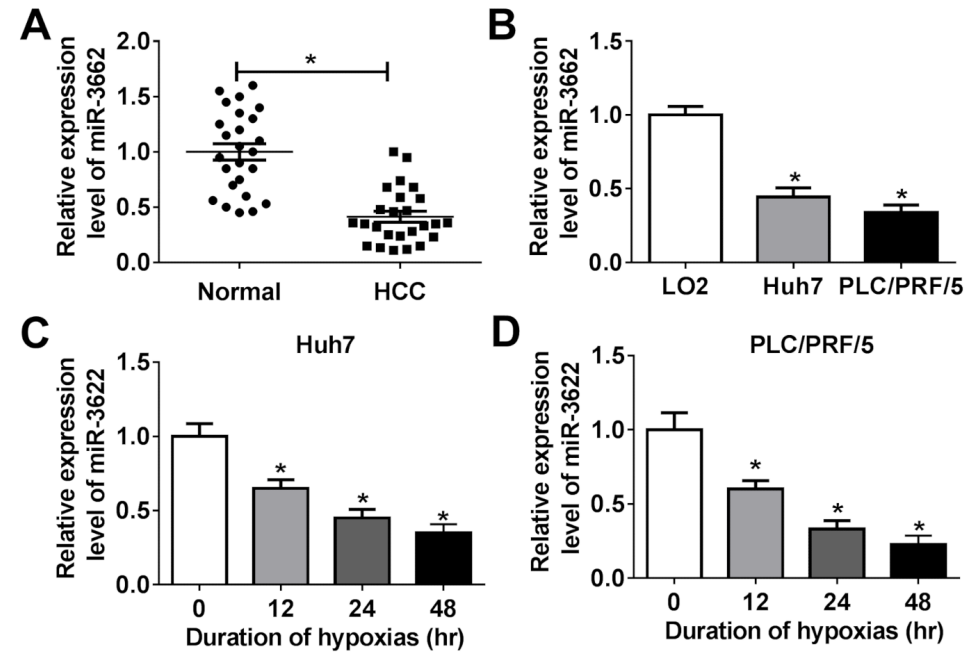

Figure 1. The expression of miR-3662 was downregulated in HCC tissues, HCC cells, and hypoxia-induced HCC cells. A, B) The expression level of miR-3662 was detected in tissues (A) and cells (B) by qRT-PCR. C, D) The expression of miR-3662 in hypoxia-induced Huh7 (C) and PLC/PRF/5 (D) cells was measured using $\mathrm{qRT}$-PCR. ${ }^{*} \mathrm{p}<0.05$ 
in HCC tissues. Similarly, miR-3662 expression was also reduced in Huh7 and PLC/PRF/5 cells compared with that in $\mathrm{LO} 2$ cells (Figure 1B). To investigate whether hypoxia regulated miR-3662 expression, Huh7 and PLC/PRF/5 cells were exposed to $1 \% \mathrm{O}_{2}$. Then, we found that the expression of miR-3662 was significantly downregulated in hypoxiainduced Huh7 and PLC/PRF/5 cells in a time-dependent manner (Figures 1C, 1D). These data revealed that the expression of miR-3662 was significantly decreased in HCC tissues and cells, and it was reduced in hypoxia-induced HCC cells in a time-dependent manner.

Overexpression of miR-3662 inhibited cell proliferation, invasion, and glucose metabolism in HCC cells. To further explore the effect of miR-3662 in HCC, miR-3662 or miR-NC was transfected into Huh7 and PLC/PRF/5 cells. As displayed in Figure 2A, the transfection of miR-3662 led to a significant increase of miR-3662 expression in Huh7 and PLC/PRF/5 cells. CCK-8 assay suggested that the proliferation of Huh7 and PLC/PRF/5 cells were both markedly inhibited at different time points in the miR-3662 group relative to the miR-NC group (Figures $2 \mathrm{~B}, 2 \mathrm{C}$ ). In addition, transwell assay suggested that the addition of miR-3662 obviously repressed the invasion ability of Huh7 and PLC/ PRF/5 cells (Figure 2D). Besides, overexpression miR-3662 evidently reduced glucose consumption and lactate production (Figures 2E, 2F). Taken together, these findings showed that miR-3662 suppressed cell proliferation, invasion, and glucose metabolism in HCC cells.

HK2 was a direct target of miR-3662 in HCC cells, and HK2 expression was increased in hypoxia-induced HCC cells. Since HK2 could promote tumor growth, we tried to investigate whether HK was a potential target for miR-3662 in HCC. The potential binding sites of miR-3662 and TRAF3 were predicted by TargetScan, suggesting the potential relationship between miR-3662 and HK2 (Figure 3A). Then the prediction was confirmed by luciferase reporter assay. The result showed that transfection of miR-3662 resulted in a significant reduction in relative luciferase activity of HK2-3'UTR-WT reporter, but not that of MUT-PVT1 reporter in Huh7 and PLC/PRF/5 cells (Figures 3B, 3C). In addition, it was shown that the protein level of $\mathrm{HK} 2 / \beta$-actin was markedly elevated in hypoxia-induced Huh7 and PLC/ $\mathrm{PRF} / 5$ cells (Figure 3D). To further explore the relationship between miR-3662 and HK2, Huh7, and PLC/PRF/5 cells
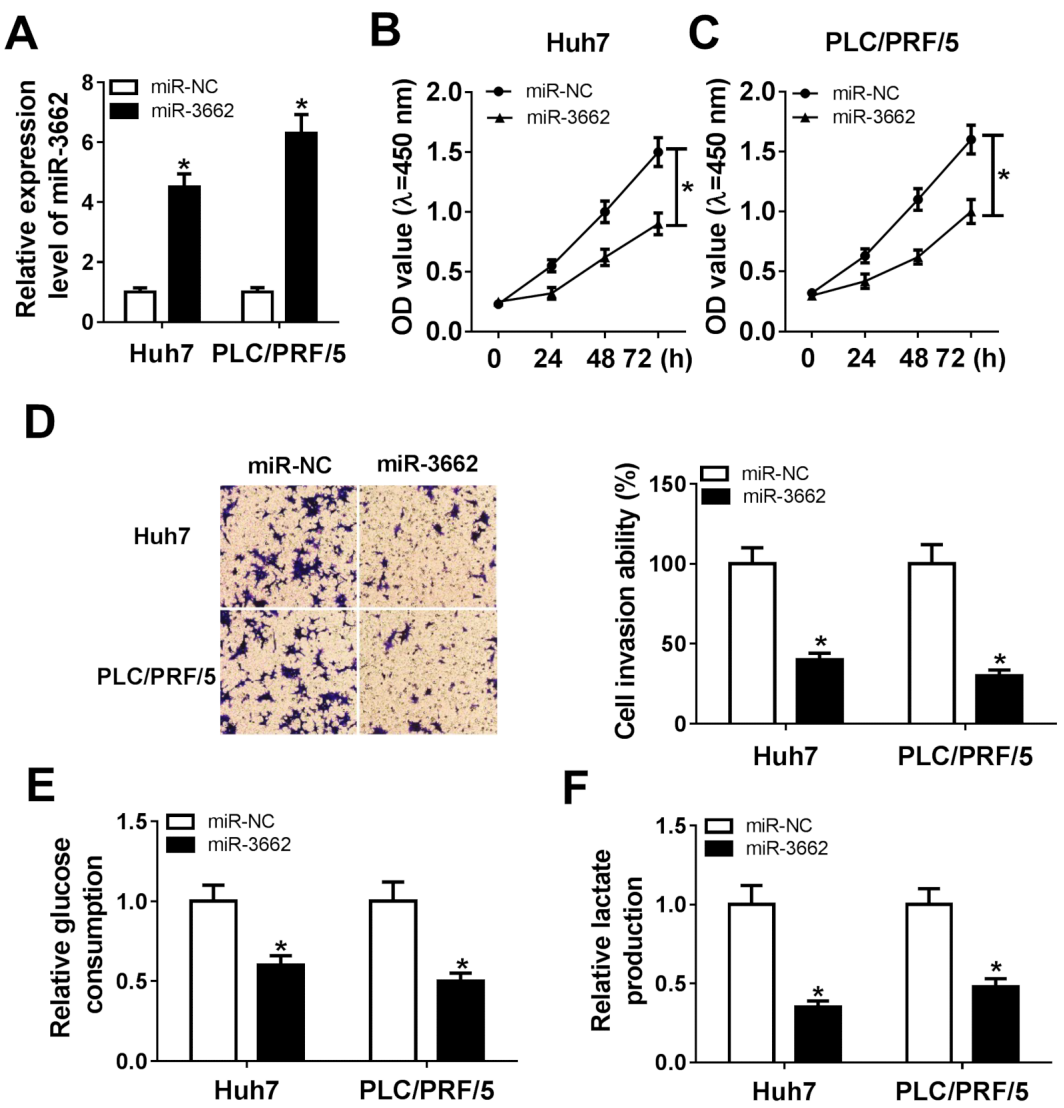

Figure 2. Overexpression of miR-3662 inhibited cell proliferation, invasion, and glucose metabolism in HCC cells. Huh7 and PLC/PRF/5 cells were transfected with miR-3662 or miR-NC. A) The expression of miR-3662 was measured in transfected Huh7 and PLC/PRF/5 cells by qRT-PCR. B, C) The effects of miR-3662 on cell proliferation were examined in Huh7 and PLC/PRF/5 cells by CCK-8 assay. D) Cell invasion was detected via transwell assay. E, F) Glucose consumption and lactate production assays were used to detect glucose metabolism activity in $\mathrm{Huh} 7$ and PLC/PRF/5 cells. ${ }^{*} \mathrm{p}<0.05$ 
A

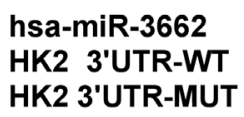

B
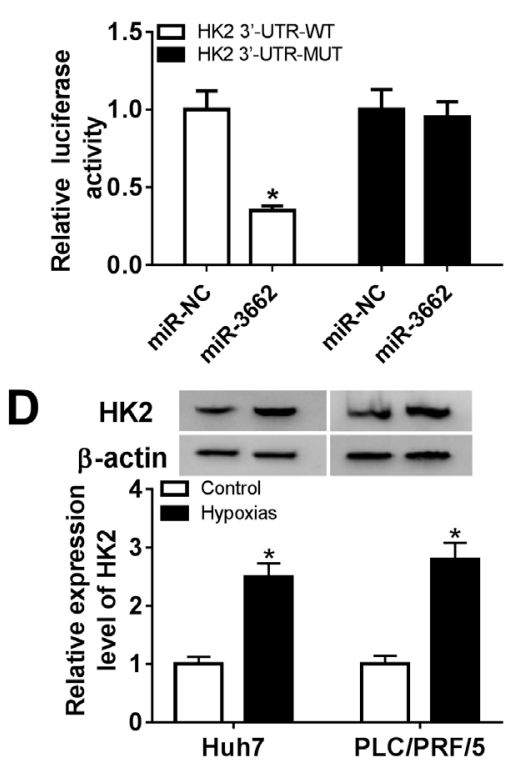

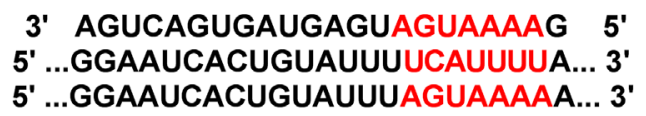

C
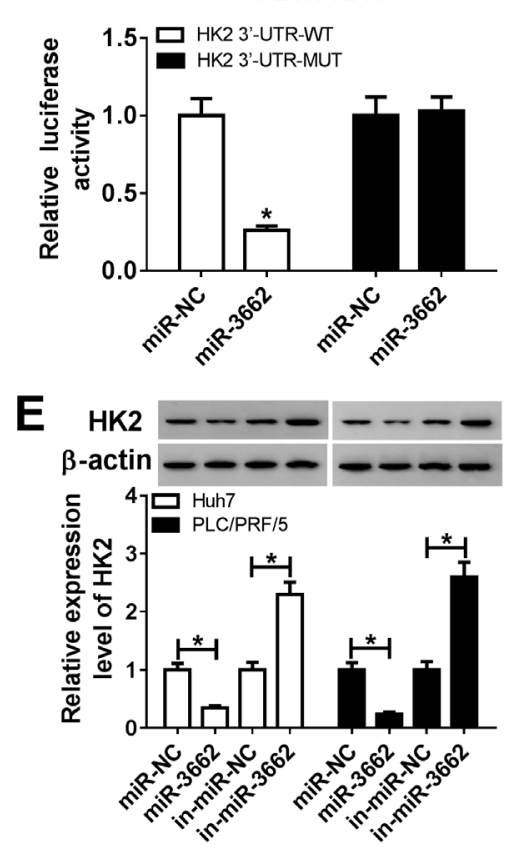

Figure 3. HK2 was a direct target of miR-3662 in HCC cells and HK2 expression was increased in hypoxia-induced HCC cells. A) The possible binding sites of miR-3662 and HK2 were predicted by TargetScan Tools. B, C) Huh7 and PLC/PRF/5 cells were co-transfected with HK2-3'UTR-WT or HK2-3'UTR-MUT reporter and miR-3662 or miR-NC, followed by the detection of relative luciferase activity. D) The expression of HK2 protein was measured in hypoxia-induced Huh7 and PLC/PRF/5 cells by western blot. E) The protein expression of HK2 was analyzed in Huh7 and PLC/PRF/5 cells transfected with miR-3662, in-miR-3662 or their match control by western blot assay. ${ }^{\star} \mathrm{p}<0.05$

were transfected with miR-3662, in-miR-3662 or their match control. As shown in Figure 3E, the transfection of miR-3662 suppressed $\mathrm{HK} 2 / \beta$-actin protein level and its knockdown showed an opposite effect in Huh7 and PLC/PRF/5 cells. Our data suggested that HK2 was a target of miR-3662, and miR-3662 might negatively regulate HK2 in Huh7 and PLC/ PRF/5 cells.

Knockdown of $\mathrm{HK} 2$ inhibited cell proliferation, invasion, and glucose metabolism in HCC cells. To investigate the effect of HK2 on HCC progression, si-HK2 or sh-NC was transfected into Huh7 and PLC/PRF/5 cells. As displayed in Figure 4A, western blot analysis suggested the transfection of si-HK2 dramatically inhibited the protein expression of the $\mathrm{HK} 2 / \beta$-actin versus the sh-NC control group. It was indicated from CCK-8 assay that, compare with the sh-NC group, the proliferation of Huh7 and PLC/PRF/5 cells were both significantly inhibited in a time-dependent manner in the si-HK2 group (Figures $4 \mathrm{~B}, 4 \mathrm{C}$ ). The invasive ability of Huh7 and PLC/PRF/5 cells was evaluated by transwell invasion assay. Similarly, the downregulation of HK2 dramat- ically suppressed the invasion ability of Huh7 and PLC/ PRF/5 cells (Figure 4D). Measurement of metabolic parameters showed that the glucose uptake and the lactate production were significantly decreased in Huh7 and PLC/PRF/5 cells with HK2 knockdown (Figures 4E, 4F). These findings revealed that knockdown of $\mathrm{HK} 2$ inhibited cell proliferation, invasion, and glucose metabolism in HCC cells.

HK2 upregulation reversed the effects of miR-3662 overexpression on proliferation, invasion and glucose metabolism in HCC cells. To further explore the relationship between HK2 and miR-3662 in HCC cells, miR-3662, miR-NC, miR-3662+HK2 or miR-3662 + pcDNA was transfected into Huh7 and PLC/PRF/5 cells. Then the effect of miR-3662 on HK2 expression was investigated in Huh7 and PLC/PRF/5 cells. Western blot analysis displayed that overexpression miR-3662 resulted in a notable reduction of $\mathrm{HK} 2 / \beta$-actin level, whereas it was ablated by the addition of HK2 in Huh7 and PLC/PRF/5 cells (Figure 5A). Furthermore, the effects of co-transfection with miR-3662 and $\mathrm{HK} 2$ on cell proliferation and invasion were investigated. 

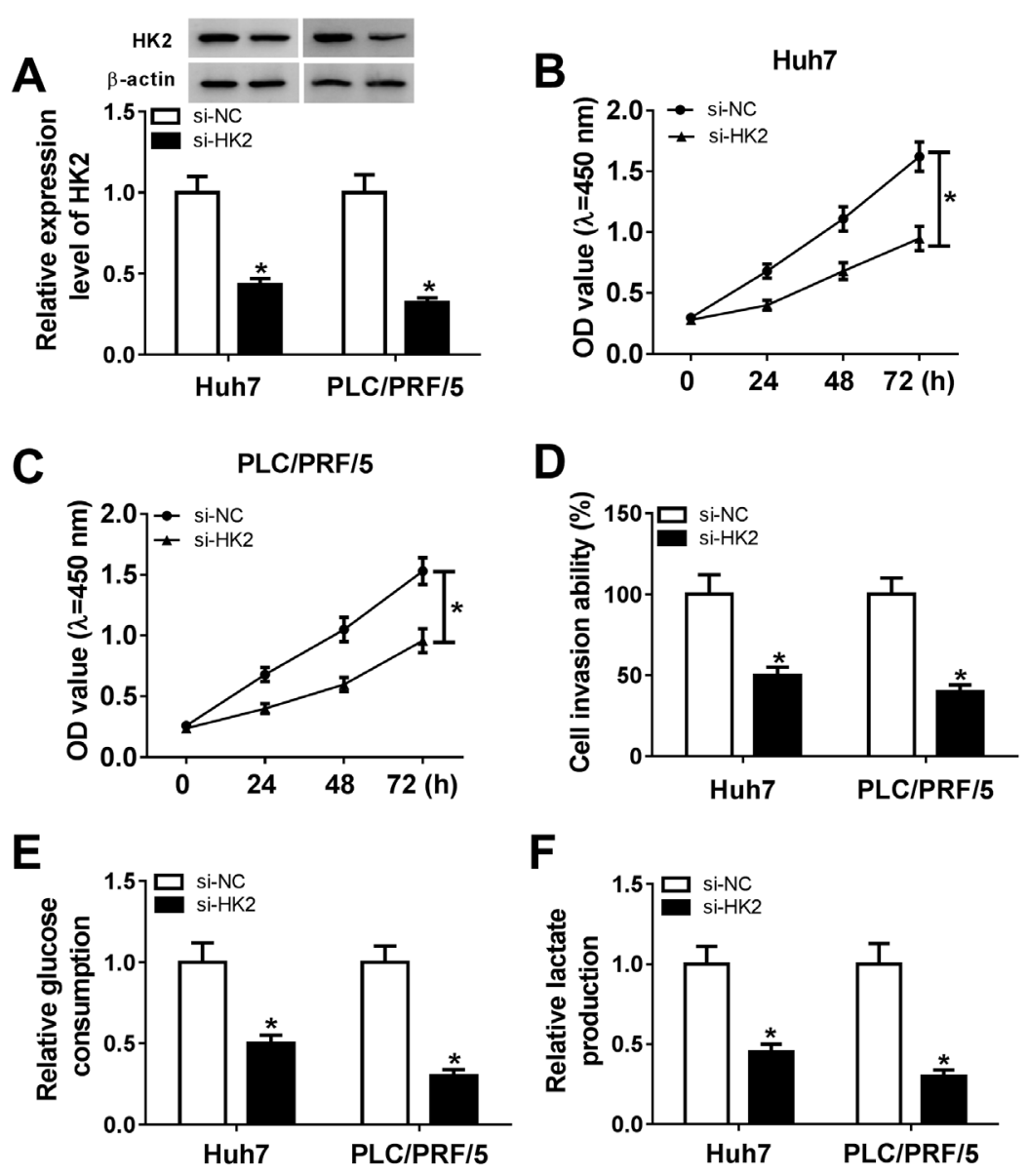

Figure 4. The knockdown of HK2 inhibited cell proliferation, invasion, and glucose metabolism in HCC cells. Huh7 and PLC/PRF/5 cells were transfected with si-HK2 or si-NC. A) The protein expression of HK2 was analyzed in Huh7 and PLC/PRF/5 cells by western blot assay. B, C) The cell proliferation of transfected cells was examined by CCK-8 assay. D) Transwell assay was employed to evaluate cell invasion in transfected Huh7 and PLC/PRF/5 cells. E, F) Glucose consumption and lactate production assays were used to detect glucose metabolism activity in Huh7 and PLC/PRF/5 cells. ${ }^{\star} \mathbf{p}<0.05$

As presented in Figures 5B-5D, transfection of miR-3662 significantly suppressed proliferation and invasion ability of Huh7 and PLC/PRF/5 cells, whereas overexpression of HK2 abolished these effects. To assess whether the addition of HK2 could reverse the effect of overexpression of miR-3662 on glucose uptake and lactate production, we measured glucose consumption and lactate production in Huh7 and $\mathrm{PLC} / \mathrm{PRF} / 5$ cells. As expected, overexpression of miR-3662 led to an obvious reduction of glucose consumption and lactate production, which was abrogated by the transfection of HK2 (Figures 5E, 5F). Our data suggested that miR-3662 exerted functional effects by targeting HK2 in HCC cells.

Transfection of HK2 could abolish the effects of miR-3662 overexpression on tumor growth and glucose metabolism in vivo. To explore the effects of miR-3662 and HK2 on HCC in vivo, Huh7 cells transfected with miR-3662, HK2 or miR-3662+HK2 were subcutaneously injected into nude mice. Forced expression of miR-3662 obviously inhibited tumor volume and weight compared with the control group, overexpression of HK2 resulted in a significant increase of tumor volume and weight, but transfection of miR-3662+HK2 had no evident influence on tumor growth (Figures 6A, 6B). Similarly, glucose consumption and lactate production were prominently limited in tumor transfected with miR-3662, while overexpression of HK2 showed opposite effects, but transfection of miR-3662+HK2 also had no evident influence on glucose metabolism (Figures 6C, 6D). Collectively, these results disclosed that the transfection of HK2 could reverse the effects of miR-3662 overexpression on tumor growth proliferation and glucose metabolism in vivo.

\section{Discussion}

HCC, which is one of the most common malignancies and has high mortality rates, is the most common type of primary liver cancer around the world [21]. It has been widely recognized that miRNAs have the potential possibility of serving as 

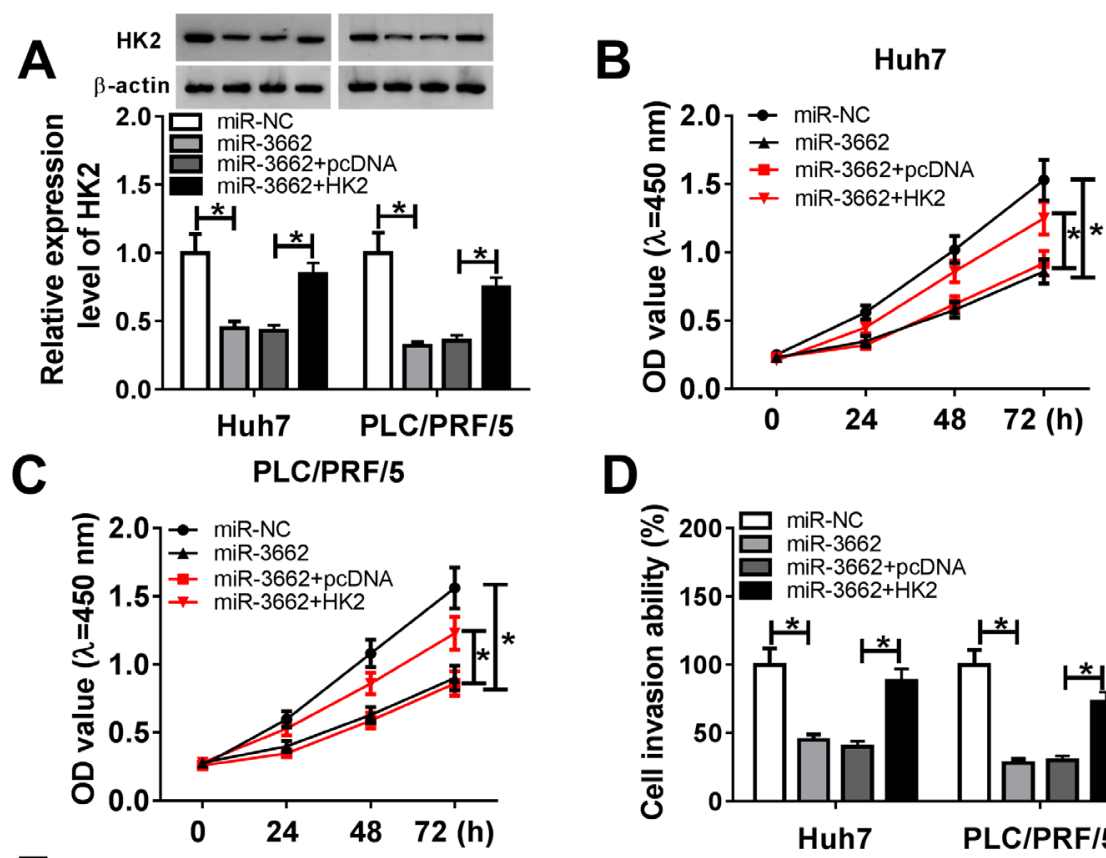

D
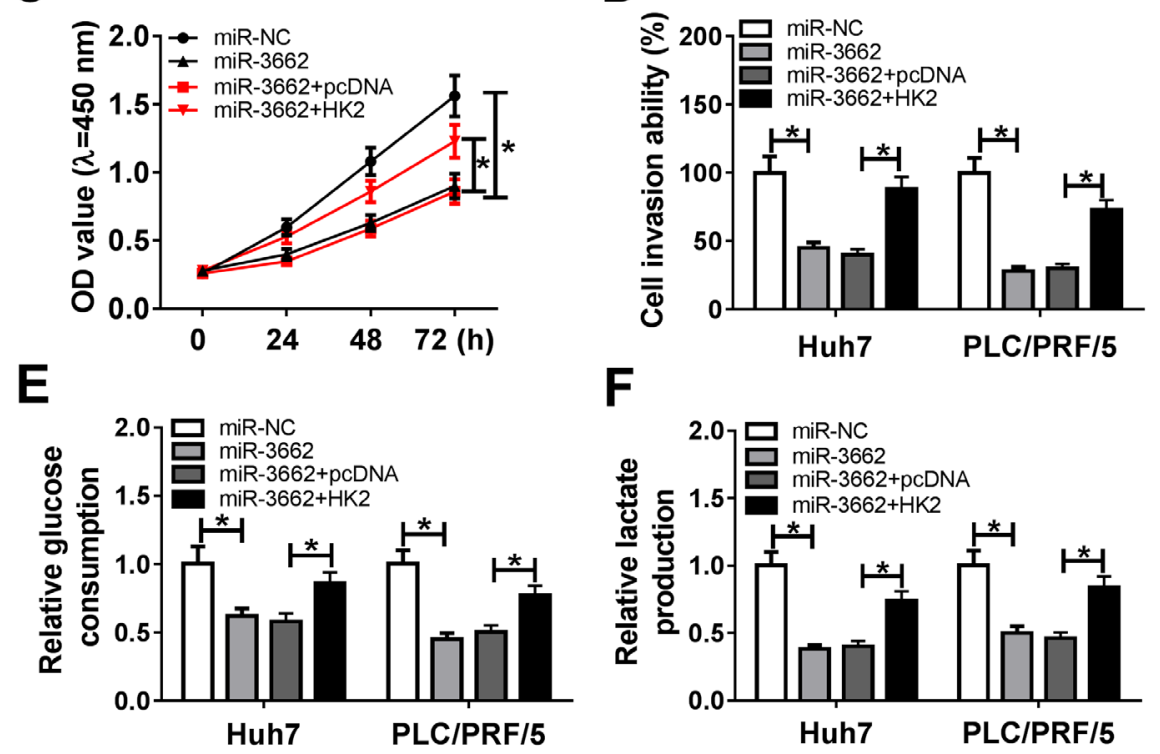

Figure 5. HK2 overexpression reversed miR-3662-mediated inhibited progression of HCC. Huh7 and PLC/PRF/5 cells were transfected with miR-3662, miR-NC, miR-3662+HK2 or miR-3662+pcDNA. A) The protein expression of HK2 was analyzed in Huh7 and PLC/PRF/5 cells by western blot assay. B, C) CCK-8 assay was conducted to analyze the cell proliferation of transfected Huh7 and PLC/PRF/5 cells. D) Cell invasion was examined by transwell assay. E, F) Glucose consumption and lactate production assays were used to detect glucose metabolism activity in Huh7 and PLC/PRF/5 cells. ${ }^{\star} \mathrm{p}<0.05$

new therapeutic strategies for cancers because they can act as oncogenes or tumor suppressors and be involved in various physiological and pathological processes [22]. Therefore, it is particularly important to understand more about the underlying mechanisms of miRNAs for the treatment of HCC.

Recently, miR-3662 has been proven to be frequently dysregulated in various diseases and has multiple functions in a wide range of biological processes. For example, acting as a tumor suppressor, overexpression of miR-3662 has anti-leukemic effects by targeting IKK $\beta$ [23]. Besides, the miR-3662 expression level is upregulated in patients with lung cancer compared with healthy controls [24]. Interestingly, these findings suggested that miR-3662 expression is decreased in some types of tumors, but increased in others. Although it had been reported that miR-3662 suppressed HCC growth by inhibition of HIF-1a-mediated Warburg effect, the specific mechanisms underlying miR-3662 remain largely unknown in the progression of HCC. In our study, we demonstrated that the expression of miR-3662 was significantly reduced in HCC tissues and cells, which was in line with previous studies. It had been reported that hypoxia played an important role in the characteristic behavior of most solid tumors [25]. Here, we found that hypoxia prominently downregulated miR-3662 expression in Huh7 and $\mathrm{PLC} / \mathrm{PRF} / 5$ cells in a time-dependent manner. In addition, overexpression of miR-3662 resulted in a significant decrease in tumor growth in vivo. These data indicated that miR-3662 might act as a tumor suppressor in HCC.

Emerging evidence has suggested that miRNAs could target many different downstream effectors in different tumors. Bioinformatic analysis was used to search for the direct target of miR-3662. In our study, results revealed that HK2 contained potential binding sites of miR-3662. Subsequently, the luciferase activity analysis was used to confirm 
A

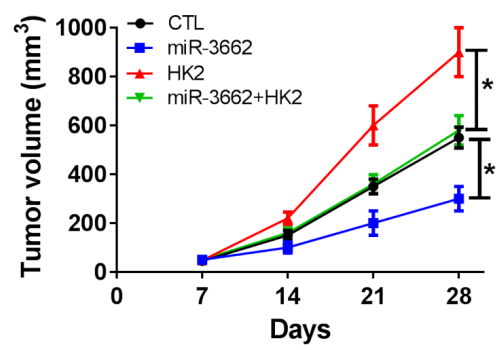

C

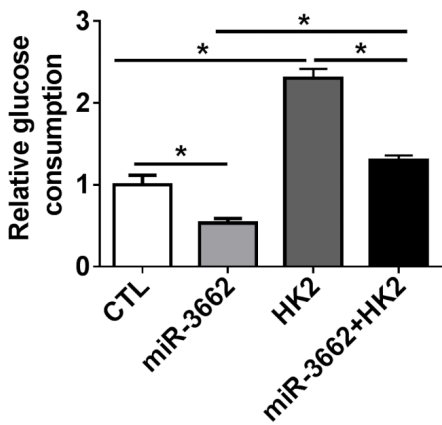

B

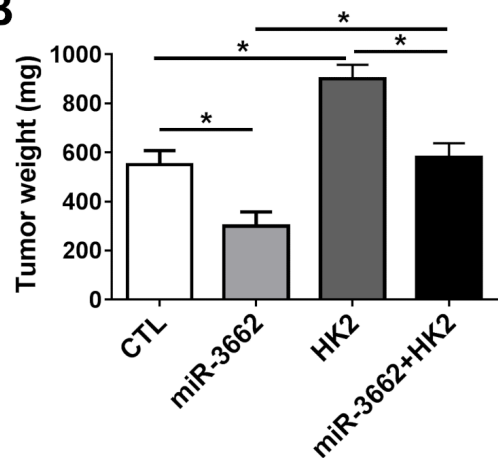

D

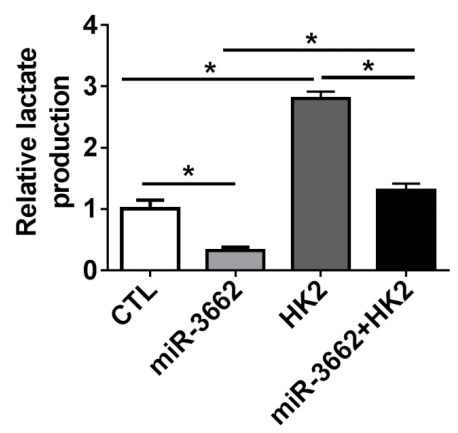

Figure 6. Transduction of HK2 could abolish the effects of miR-3662 overexpression on tumor growth and glucose metabolism in vivo. Huh7 cells transfected with miR-3662, HK2 or miR-3662+HK2 were injected subcutaneously into nude mice. A) Tumors volume were examined every 7 d. B) The resected tumors were weighed at $28 \mathrm{~d}$ post-injection. $\mathrm{C}, \mathrm{D})$ Glucose consumption and lactate production assays were used to detect glucose metabolism activity in tumor tissues. ${ }^{\star} \mathrm{p}<0.05$

the prediction. Here, we first identified HK2 as a direct target of miR-3662. In general, hypoxic conditions induced an increase in tumor aggressiveness, stimulate epithelial-mesenchymal transition and resulted in the activation of cancer cell autonomous growth $[26,27]$. The increased tolerance of cancer cells to hypoxia is based on the different factorsfrom increase of glycolysis (known as the Warburg Effect) to activate intracellular growth pathways [28]. HK2 is one of the critical metabolic enzymes and highly expressed in various types of cancer cells. For instance, the expression of HK2 was markedly elevated in colorectal carcinoma tissues relative to normal tissues [29]. Moreover, the mRNA and protein levels of HK2 in ovarian cancer tissues were significantly higher than those in normal ovarian tissues [30]. The previous study had identified that the protein level of HK2 was upregulated in the HCC tissues [31]. Consistent with the previous study, we found that the protein level of HK2 was markedly elevated in Huh7 and PLC/PRF/5 cells. In addition, knockdown of HK2 inhibited cell proliferation, invasion, and glucose metabolism in Huh7 and PLC/PRF/5 cells. Besides, overexpression of $\mathrm{HK} 2$ reversed miR-3662-mediated repressed progression of HCC. Moreover, our data demonstrated that transfection of HK2 abolished inhibitory effects of miR-3662 overexpression on tumor growth and glucose metabolism in vivo. Thus, these data indicated that miR-3662 could regulate cell growth, invasion, and glucose metabolism by targeting HK2 in HCC cells.

In conclusion, the expression of miR-3662 was downregulated in HCC tissues and cells. Upregulation of miR-3662 inhibited cell proliferation, invasion and glucose metabolism in HCC cells, which was reversed by the addition of HK2. In addition, the transfection of HK2 also abolished the effects of miR-3662 overexpression on tumor growth and glucose metabolism in vivo. Collectively, miR-3662 regulated cell growth, invasion, and glucose metabolism by targeting HK2 in HCC, providing a promising avenue for treatment of HCC. Besides, the miR-3662/HK2 axis might provide a possible new strategy for the treatment of other cancers in the future.

\section{References}

[1] VENOOK AP, PAPANDREOU C, FURUSE J, DE GUEVARA LL. The incidence and epidemiology of hepatocellular carcinoma: a global and regional perspective. Oncologist 2010; 15: 5-13. https://doi.org/10.1634/theoncologist.2010S4-05

[2] FORNER A, LLOVET JM, BRUIX J. Hepatocellular carcinoma. Lancet 2012; 379: 1245-1255. https://doi.org/10.1016/ S0140-6736(11)61347-0 
[3] ROSSI L, ZORATTO F, PAPA A, IODICE F, MINOZZI M et al. Current approach in the treatment of hepatocellular carcinoma. World J Gastrointest Oncol 2010; 2: 348-359. https:// doi.org/10.4251/wjgo.v2.i9.348

[4] SUBRAMANIAN S, FU Y, SUNKAR R, BARBAZUK WB, ZHU JK et al. Novel and nodulation-regulated microRNAs in soybean roots. BMC Genomics 2008; 9: 160. https://doi. org/10.1186/1471-2164-9-160.

[5] AMBROS V. The functions of animal microRNAs. Nature 2004; 431: 350-355. https://doi.org/10.1038/nature02871

[6] AURORA EK, SLACK FJ. Oncomirs - microRNAs with a role in cancer. Nat Rev Cancer 2006; 6: 259-269. https://doi. org/10.1038/nrc1840

[7] LEIGH-ANN M, MURPHY PR. MicroRNA: Biogenesis, Function and Role in Cancer. Curr Genomics 2010; 11: 537561. https://doi.org/10.2174/138920210793175895

[8] HUANG S, HE X. The role of microRNAs in liver cancer progression. Br J Cancer 2011; 104: 235-240. https://doi. org/10.1038/sj.bjc.6606010

[9] MAHARRY SE, WALKER CJ, LIYANARACHCHI S, MEHTA S, PATEL M et al. Dissection of the Major Hematopoietic Quantitative Trait Locus in Chromosome 6q23.3 Identifies miR-3662 as a Player in Hematopoiesis and Acute Myeloid Leukemia. Cancer Discov 2016; 6: 1036-1051. https://doi. org/10.1158/2159-8290.CD-16-0023

[10] CHEN Z, ZUO X, ZHANG Y, HAN G, ZHANG L et al. MiR3662 suppresses hepatocellular carcinoma growth through inhibition of HIF-1 1 -mediated Warburg effect. Cell Death Dis 2018; 9: 549. https://doi.org/10.1038/s41419-018-0616-8

[11] WILSON WR, HAY MP. Targeting hypoxia in cancer therapy. Nat Rev Cancer 2011; 11: 393-410. https://doi. org/10.1038/nrc3064

[12] COURTNAY R, NGO DC, MALIK N, VERVERIS K, TORTORELLA SM et al. Cancer metabolism and the Warburg effect: the role of HIF-1 and PI3K. Mol Biol Rep 2015; 42: 841-851. https://doi.org/10.1007/s11033-015-3858-x

[13] WARBURG O. On the origin of cancer cells. Science 1956; 123: 309-314. https://doi.org/10.1126/science.123.3191.309

[14] KOPPENOL WH, BOUNDS PL, DANG CV. Otto Warburg's contributions to current concepts of cancer metabolism. Nat Rev Cancer 2011; 11: 325-337. https://doi.org/10.1038/ nrc3038

[15] WARD PS, THOMPSON CB. Metabolic Reprogramming: A Cancer Hallmark Even Warburg Did Not Anticipate. Cancer Cell 2012; 21: 297-308. https://doi.org/10.1016/j. ccr.2012.02.014

[16] WILSON JE. Isozymes of mammalian hexokinase: structure, subcellular localization and metabolic function. J Exp Biol 2003; 206: 2049-2057. https://doi.org/10.1242/jeb.00241

[17] LYSHCHIK A, HIGASHI T, HARA T, NAKAMOTO Y, FUJIMOTO $\mathrm{K}$ et al. Expression of glucose transporter-1, hexokinase-II, proliferating cell nuclear antigen and survival of patients with pancreatic cancer. Cancer Invest 2007; 25: 154-162. https://doi.org/10.1080/07357900701208931

[18] RHO M, KIM J, JEE CD, LEE YM, LEE HE et al. Expression of Type 2 Hexokinase and Mitochondria-related Genes in Gastric Carcinoma Tissues and Cell Lines. Anticancer Res 2007; 27: 251-258.
[19] CHEN J, ZHANG S, LI Y, TANG Z, KONG W. Hexokinase 2 overexpression promotes the proliferation and survival of laryngeal squamous cell carcinoma. Tumour Biol 2014; 35: 3743-3753. https://doi.org/10.1007/s13277-013-1496-2

[20] KWEE SA, HERNANDEZ B, CHAN O, WONG L. Choline kinase alpha and hexokinase-2 protein expression in hepatocellular carcinoma: association with survival. PLoS One 2012; 7: e46591. https://doi.org/10.1371/journal.pone.0046591

[21] TORRE LA, BRAY F, SIEGEL RL, FERLAY J, LORTETTIEULENT J et al. Global cancer statistics, 2012. CA Cancer J Clin 2015; 65: 87-108. https://doi.org/10.3322/caac.21262

[22] ZHANG B, PAN X, COBB GP, ANDERSON TA. microRNAs as oncogenes and tumor suppressors. Dev Biol 2007; 302: 1-12. https://doi.org/10.1016/j.ydbio.2006.08.028

[23] MAHARRY SE, WALKER CJ, LIYANARACHCHI S, MEHTA S, PATEL M et al. Dissection of the major hematopoietic quantitative trait locus in chromosome 6q23.3 identifies miR-3662 as a novel player in hematopoiesis and acute myeloid leukemia. Cancer Discov 2016; 6: 1036-1051. https:// doi.org/10.1158/2159-8290.CD-16-0023

[24] POWRÓZEK T, KRAWCZYK P, KOWALSKI DM, WINIARCZYK K, OLSZYNA-SEREMENTA $M$ et al. Plasma circulating microRNA-944 and microRNA-3662 as potential histologic type-specific early lung cancer biomarkers. Transl Res 2015; 166: 315-323. https://doi.org/10.1016/j. trsl.2015.05.009

[25] BABU G. Hypoxia and Cancer. Wilderness \& Environmental Medicine 2015; 26: e3-e4. https://doi.org/10.1016/j. wem.2014.11.015

[26] AXELSON H, FREDLUND E, OVENBERGER M, LANDBERG G, PÅHLMAN S. Hypoxia-induced dedifferentiation of tumor cells - A mechanism behind heterogeneity and aggressiveness of solid tumors. Semin Cell Dev Biol 2005; 16: 554-563. https://doi.org/10.1016/j.semcdb.2005.03.007

[27] VAUPEL P. The Role of Hypoxia-Induced Factors in Tumor Progression. Oncologist 2004; 9: 10-17. https://doi. org/10.1634/theoncologist.9-90005-10

[28] SCHERBAKOV AM, STEFANOVA LB, SOROKIN DV, SEMINA SE, BERSTEIN LM et al. Snail/beta-catenin signaling protects breast cancer cells from hypoxia attack. Exp Cell Res 2013; 319: 3150-3159. https://doi.org/10.1016/j. yexcr.2013.08.019

[29] ZHU W, HUANG Y, PAN Q, XIANG P, XIE N et al. MicroRNA-98 Suppress Warburg Effect by Targeting HK2 in Colon Cancer Cells. Dig Dis Sci 2017; 62: 660-668. https:// doi.org/10.1007/s10620-016-4418-5

[30] ZHANG XY, ZHANG M, CONG Q, ZHANG MX, ZHANG MY et al. Hexokinase 2 confers resistance to cisplatin in ovarian cancer cells by enhancing cisplatin-induced autophagy. Int J Biochem Cell Biol 2018; 95: 9-16. https://doi. org/10.1016/j.biocel.2017.12.010

[31] JIN F, WANG Y, ZHU Y, LI S, LIU Y et al. The miR-125a/ HK2 axis regulates cancer cell energy metabolism reprogramming in hepatocellular carcinoma. Sci Rep 2017; 7: 3089. https://doi.org/10.1038/s41598-017-03407-3 